\title{
SymmeGuess: Fun beyond Symmetry Learning
}

\author{
Lee-Loon Ong ${ }^{2}$, Ah-Choo Koo ${ }^{1}$, Yi-Fei Tan ${ }^{2}$, Wooi-Nee Tan ${ }^{2}$, Forest Yan-Peng Lim ${ }^{1}$ \\ ${ }^{1}$ Faculty of Creative Multimedia \\ ${ }^{2}$ Faculty of Engineering, Multimedia University, Cyberjaya, Selangor, Malaysia. \\ E-mail:lelon81@hotmail.com,ackoo@mmu.edu.my,yftan@mmu.edu.my,wntan@mmu.edu.my,forest.lim@mmu.edu.my
}

\begin{abstract}
Computer application can be used for artistic design and as a means to facilitate the understanding of mathematical concepts. This paper presents a game-based application as a visual learning tool for mathematics and art subjects. The objectives of this application are to enable learners to build their knowledge on symmetry and to explore the beauty of symmetrical patterns with confidence and enjoyment.
\end{abstract}

Keywords — geometry; symmetrical pattern; game based learning

\section{Problem Statement And Purpose}

"Knowledge of many of the geometric principles, concepts and perspectives underlying structure and form in design can be sourced in ancient times." [1]. Applications of geometry had been explored much by Euclid (c.300 BCE) and many other scholars such as M. C. Escher and Felix Klein. Now, geometry is still a useful knowledge for many professionals to build up their understanding of scientific and artistic knowledge for their professions. The foundations of such knowledge have been largely absent from the design of curriculum worldwide, and there is a lack of exploration and appreciation of geometry knowledge as a research tool [1]. This lacking should be a main concern in mathematics curriculum and more emphasis must be placed on innovative ways of approaching the teaching and learning of geometry in classrooms.

In this paper, the concept of symmetry is explored and applied into a game-based learning application, namely SymmeGuess. By playing the game, users are able to learn fundamental concepts of symmetry, and at the same time to connect the knowledge of symmetry with visually rich artistic symmetrical pattern.

\section{LITERATURE REVIEW}

\section{A. Symmetry Group and Rosette Pattern}

Symmetry is an important concept in both art and mathematics. It is described as a harmony or beautiful form because of its balanced proportions. Many contend that symmetrical objects, such as faces or body structure of living things are inherently more beautiful than asymmetrical ones.
Symmetry of an object is a property such that the object will remain invariant under certain classes of transformation. If the object matches itself after it is being rotated around a point at certain rotational angle, then the object is said to have rotational symmetry. Whereas, an object is known to have reflectional symmetry if it remains unchanged after a reflection. For a 2-dimensional object, the axis of symmetry in reflectional symmetry is a line.

In rotational symmetry, the angle of rotation of a symmetric object is the smallest angle of rotation that preserves the object. Using degrees to describe the rotational angle is inconvenient because the precise angle is not obvious by looking at the object. Instead, the order of rotation is used to describe rotational symmetry. An object has order $n$ rotational symmetry if $1 / n$ of a complete turn leaves the object unchanged.

A symmetry group is the collection of all symmetries of a plane object, and there are two types of symmetry groups, namely cyclic $(C n)$ symmetry group and dihedral $(D n)$ symmetry group. The former refers to objects with rotational symmetry and no reflectional symmetry. If the rotation has order $n$, the group is called $C n$. The latter refers to the objects with rotational symmetry and reflection across lines. If the rotation has order $n$, there will be $n$ mirror lines and the group is called $D n$.

The cyclic and dihedral symmetry groups form the rosette symmetry groups. A pattern with rosette symmetry is known as a rosette pattern [2][3]. One of the forms of rosette pattern is a round, stylized flower design, used extensively in sculptural objects from antiquity. It is derived from the natural shape of a rosette in botany, formed by leaves radiating out from the stem of a plant and visible even 
after the flowers wither. It has been used as architectural and sculptural decoration for millennia.

\section{B. Explore Symmetry through Playing}

Pierre van Hiele (1999) writes, "For children, geometry begins with play". He goes on to say that for students to reach the higher levels of geometric thinking, their instruction should still begin with "an exploratory phase, gradually building concepts and related language, and culminating in activities that help students integrate what they have learned into what they already know" [5].

The developed game-based application, SymmeGuess offers activities that can supplement geometry instruction when guiding students through exploring, building concepts, and integrating their learning through application. In this context, activity in game pushes students to explore the connection of different concepts such as reflection, order of rotation, angle of rotation from the patterns generated in the application.. The aim of this application is to expose students to mathematical concepts of symmetry, which in return are able to improve their mathematics knowledge.

\section{METHODOLOGY}

\section{A. Plan for Guess Contest and Game Flow}

Figure 1 gives the schematic process flow of the application to generate a symmetric and asymmetric pattern. The diagram shows how symmetric concepts are integrated into artistic image creation. Figure 3 shows the work flow of the application. It begins with selecting a default seed which is the basic model to generate the pattern. This is then followed by customizing the seed, and selecting the type of pattern. After all the parameters have been selected, the animation of pattern drawing begins, in which the seed will be first drawn, followed by its transformed images, one by one in circular form based on the parameters selected. Once the animation begins, the player has to guess the total number of seeds exhibit in a rosette pattern upon its completion. In order to overcome the obstacle of the game, the player need to key in the correct number of seeds/petals before the completion of a rosette pattern drawn by the application. The shorter the time spent to guess correctly, the higher the score that one can get. The reason for using "guessing" method as a game design is to provide more opportunities for learners to test their spatial visualization ability within an allocated time, while allowing them to improve their knowledge on rosette symmetry. .. Besides that, the score obtained will be stored into database to stimulate challenges with others players on the internet.

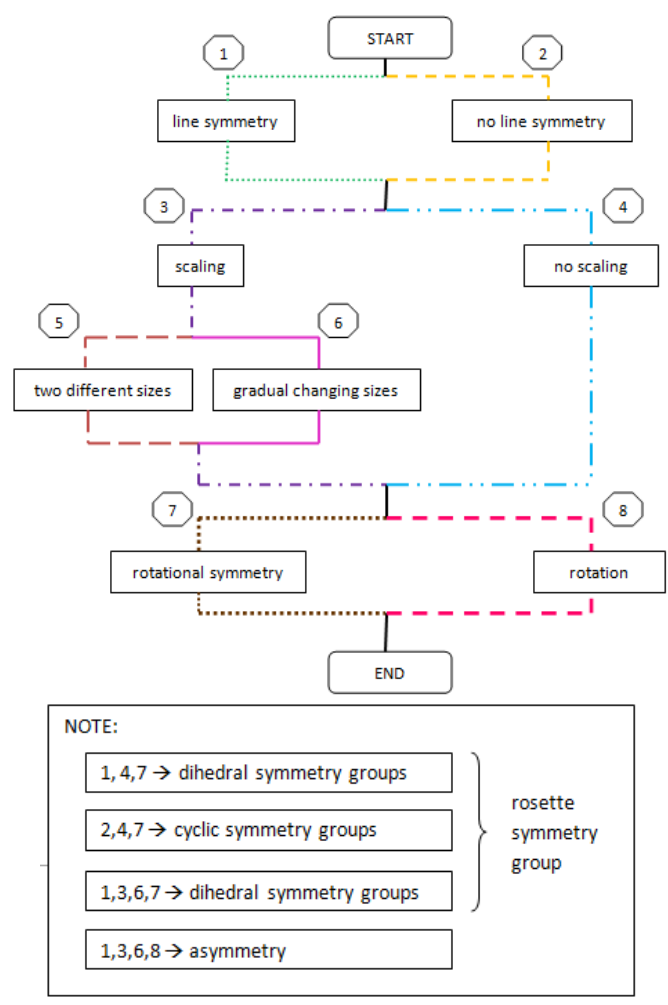

Figure 1: The schematic process flow of the application to generate a symmetric and asymmetric pattern [[Victor, the diagram has lines with different pattern of dash line, can we indicate its meaning?]]

\section{GAME DESIGN AND APPLICATION}

\section{A. Seeds for Symmetrical Pattern}

In playing the game, the player is required to choose or create a seed with line symmetry or without line symmetry (refer to Flow No 1, 2 in Figure 1). After that, the generated seed will be used as a reference. The seed can be scaled or remain unchanged. In scaling the seed, it can be made into two different sizes or changed gradually in size (refer to Flow No 5, 6 in Figure 1). Finally, different type of patterns will be generated by rotating the seeds with different order of rotation for different scaling. Figure 2 shows two examples of seed, one with line symmetry(seed A) and the other one with no line symmetry(seed B), then three patterns will be generated based on each of the seeds

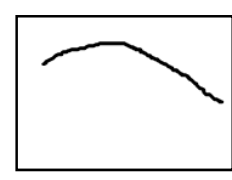

Seed Type A

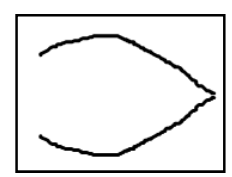

Seed Type B
Figure2: Seeds with line symmetry and no line symmetry

If the pattern is generated by rotating the seed (either seed A or B) without any changes of the size, then the pattern will have the features of rotational symmetry. Figure 4 shows two types of rosette symmetry group, the pattern generated based on Seed A is dihedral symmetry group while the pattern based on Seed B is cyclic symmetry group. Meanwhile, if two different sizes of seeds are rotated 


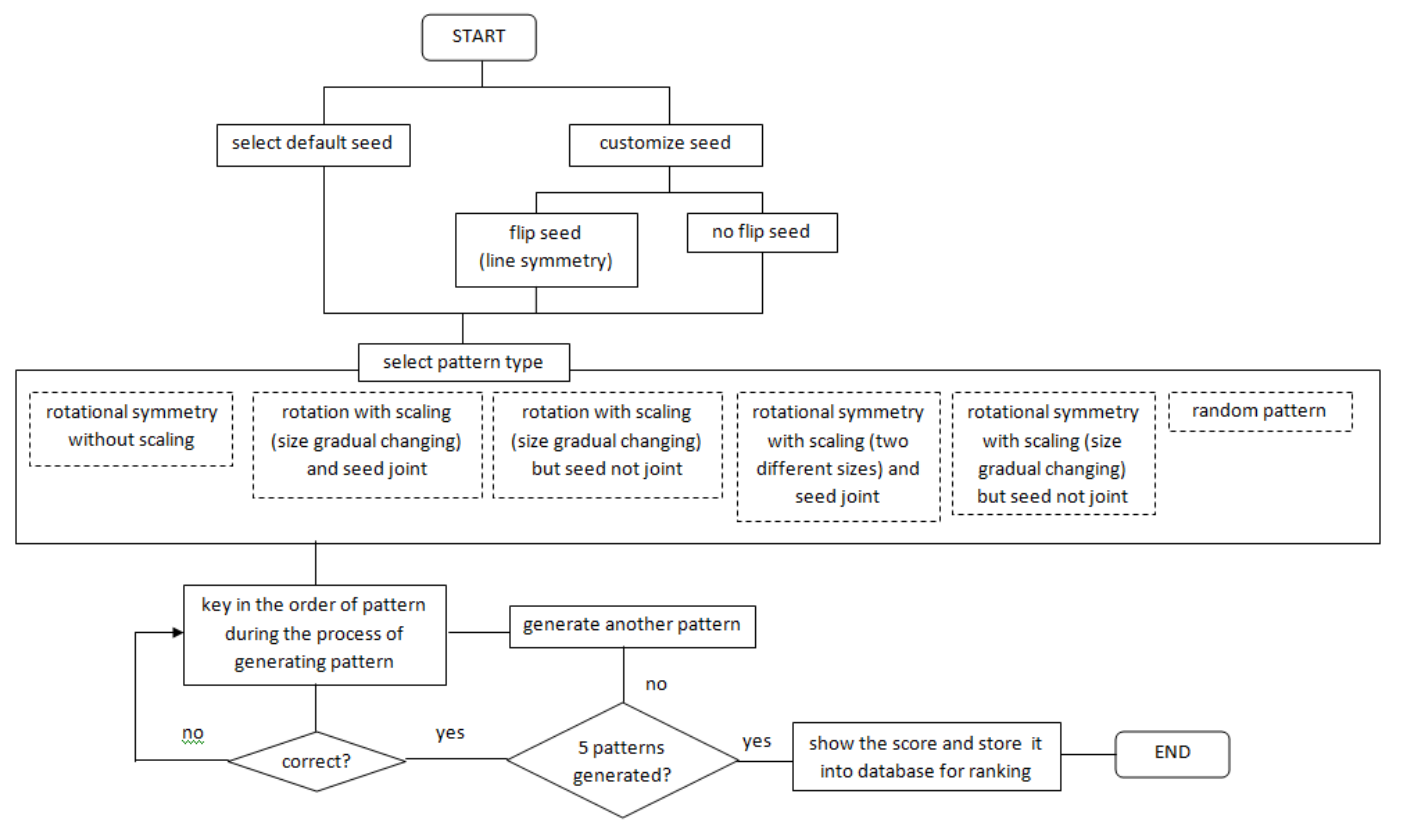

Figure 3: Game flow of the application

symmetrically, then two type of rosette symmetry patterns will be generated also, (refer to Figure 5). Lastly, if there is a gradual change in the size of the seeds and it is rotated in a non-symmetrical way, then the asymmetry patterns will be generated (refer to Figure 6).

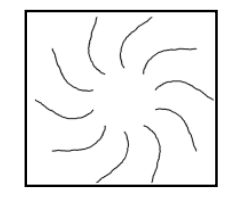

Dihedral symmetry group

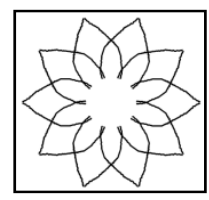

Cyclic symmetry group
Figure 4: Rosette symmetry group for same seed size

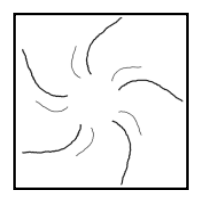

Dihedral symmetry group
Cyclic symmetry group

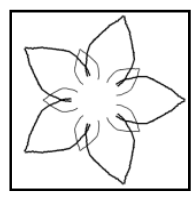

Figure 5: Rosette symmetry group for two different seed sizes
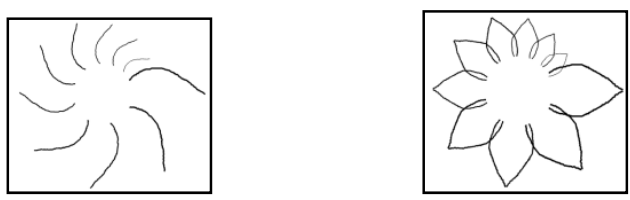

Figure 6: Asymmetry patterns for size of seeds changing gradually

\section{B. Playing SymmeGuess}

At the beginning of game, a player has to select the type of seeds. By default, there are 3 seeds with line symmetry and 2 with no line symmetry (refer to Figure 7 ). The players are also able to draw any seed that they like with different colour and thickness line. Besides that, "Flipping" feature (refer to Figure 8), enable the player to flip the drawing which determine if the seed has the reflectional symmetry or not. By adding customizable function in the game, the players can explore infinite number of pattern generated and increase the entertaining value to the game. After the seed is determined, the player is requested to select the type of scaling with rotation. In order to increase the challenging level, three other options can be chosen: (1) seed size unchanged with random radius, (2) seed size gradually change with random radius and (3) random type of scaling with random radius (refer to Figure 9).

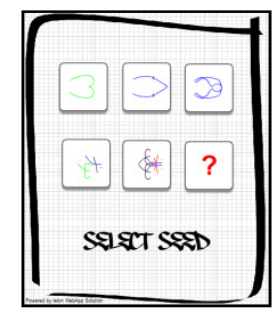

Figure 7: Select seed

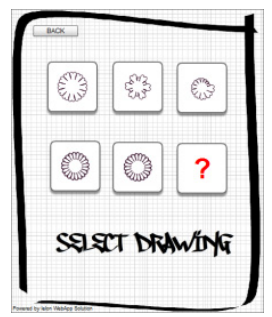

Figure 9: Select scaling and rotation type

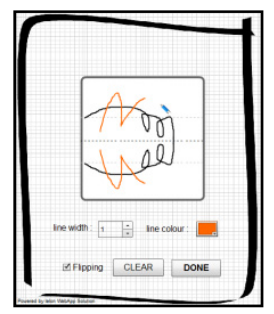

Figure 8: Draw seed

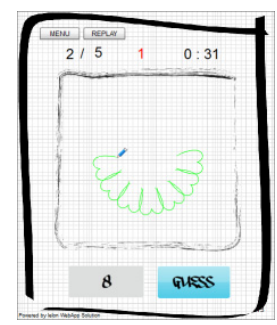

Figure 10: Guess number of seeds in total
The game is ready to start after the types of seed and scaling have been selected. When the game starts, pattern will be generated. During the process of pattern drawing, players need to key in the number of seeds (order of rotation) 
(refer to Figure 10). If the answer is wrong, the players have to guess again. On the other hand, if the answer is correct, the next pattern will be generated. The game is over if order of rotation for 5 patterns are answer correctly. At last, score, name of player and time elapsed are recorded into database for ranking purpose.

\section{Pedagogical Value of SymmeGuess}

The objective of this game is to explore fun based learning in classroom for identifying types of symmetry. According to Harrison [6], if technology is integrated into the curriculum, it will lead to learning, productivity, communication, and higher-order thinking. However, it is not technology alone that enables student learning but the pedagogical strategies designed by the teacher with the technology [7]. Therefore, a structure flow of integrating SymmeGuess into classroom instruction has been proposed, with reference to Van Hiele's [5] concept of exploration:

Step 1: Share the learning objective of the lesson with students.

Step 2: Teacher demonstrates manipulation of the application and students explore the application.

Step 3: Conduct guessing competitions. In the first round, students use default seed and in the second round, students customize the seed.

Step 4: Students observe and share the created patterns with others (refer to Figure 11). With the guidance from their teacher, students discuss with others to categorize different types of symmetry and asymmetry patterns.

Step 5: Teacher guides students to deduce types of symmetry and their characteristic.

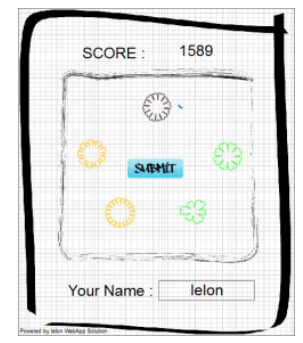

Figure 11: Observe created patterns

\section{DISCUSSION AND FUTURE WORK}

Geometrical knowledge transcends the boundary between art and mathematics. By building a substantial understanding of geometric concepts and principles, this knowledge can offer immense potential as a problem solving tool in the twenty-first century. Particularly, attention can be focused on geometric symmetry. A few recommendations for future research are described as follows:

(i) A case study should be carried out on a group of students of different ages to examine whether this application is able to build their understanding on symmetry and explore the beauty of symmetric patterns with confidence and enjoyment. (ii) This research is valuable for exploring the potential of geometry as a design tool and helping students to link up the concepts between mathematics and art. Hence, it should be extended to generate other type of pattern based on other geometric concept such as tiling and ornament.

\section{CONCLUSION}

In this paper, a game-based application was developed which enable learners to learn fundamental concept of geometry, and at the same time to explore the nature and beauty of geometrical symmetry. This paper has also highlighted the value of using computer as a tool to connect the concepts between mathematics, and visual patterns through a guessing game, called SymmeGuess.

\section{ACKNOWLEDGMENT}

This project is partially financially supported by E-science Fund (01-02-01-SF0158), MOSTI, Malaysia.

\section{REFERENCES}

[1] M.A. Hann and B.G. Thomas (2007). Beyond the Bilateral Symmetry in Two-Dimensional Design. International Association of Societies of Design Research, The Hong Kong Polytechnic University, November 2007.

[2] Anneke Bart and Bryan Clair. Introduction to symmetry. Retrieved 15 June, 2011 from http://euler.slu.edu/escher/index.php/Introduction_to_Symmetry.

[3] Horne, C.E. (2000). Geometric Symmetry in Patterns and Tilings, Woodhead Publishing, Cambridge, UK.

[4] Wikipedia. Rosette(design). Retrieved 20, June, 2011 from http://en.wikipedia.org/wiki/Rosette_(design)

[5] Van Hiele, Pierre M. (1999, February). Developing geometric thinking through activities that begin with play. Teaching Children Mathematics, 5(6), 310-316.

[6] Harrison, H. (2004). The Effectiveness of Using Technology in the Classroom: San Jose State University. Retrieved August 31, 2007 from

http://www.sjsu.edu/depts/it/harrison/Reading\%20report_188.pdf.

[7] Barnett, J. (2006). Implementation of Personal Response Units in Very Large Lecture Classes: Student Perception. Australasian Journal of Educational Technology, 22(4), 474-494. 